\title{
Síndrome de Cushing: veinticinco años de experiencia clínica en Navarra
}

\author{
Cushing's syndrome: twenty-five years of clinical experience \\ in Navarre
}

\section{Pérez García, E. Anda, J. Rojo}

\section{RESUMEN}

Fundamento. El objetivo de este trabajo es conocer la situación del Síndrome de Cushing (CS) en cuanto a etiología, diagnóstico, tratamiento y curación en los últimos 25 años en Navarra.

Material y métodos. Este estudio -retrospectivo, observacional-, se ha llevado a cabo sobre 36 pacientes diagnosticados de SC entre 1985 y 2010.

Resultados. El 80,5\% eran mujeres y el 19,5\% hombres. La edad media al diagnóstico fue $39,3 \pm 13$ años. Las principales manifestaciones clínicas fueron: obesidad (85,3\%), cara de luna llena $(35,3 \%)$ y miopatía (50\%). Etiológicamente, el $58,3 \%$ tenían origen hipofisario, el $36,1 \%$ adrenal y el 5,5\% ectópico.

La media de CLU fue $343,5 \pm 310,1 \mu \mathrm{g} / 24$ horas. El corti-

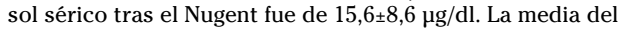
cortisol plasmático tras el frenado débil fue $15,1 \pm 14,2 \mu \mathrm{g} / \mathrm{dl}$. Existe correlación lineal directa estadísticamente significativa $(p<0,01)$ entre CLU y el Nugent, así como entre Nugent y el cortisol tras el frenado débil. No hay diferencias estadísticamente significativas entre etiología adrenal e hipofisaria en factores demográficos o resultados analíticos.

El tratamiento en todos los adenomas hipofisarios y adrenales fue quirúrgico. El $23,8 \%$ de los hipofisarios precisó una segunda cirugía y posteriormente radioterapia (RT) y el 9,5\% sólo recibió RT tras la primera intervención. En el seguimiento el $52,9 \%$ se curaron sin secuelas, el $41,1 \%$ presentan comorbilidades y el $5,9 \%$ han fallecido (sin diferencias significativas entre adrenales e hipofisarios).

Conclusiones. La etiología más frecuente del SC fue la hipofisaria, siendo más frecuente en mujeres. Todos están curados, aunque el $41,1 \%$ presentaron comorbilidades en el seguimiento.

Palabras clave. Síndrome Cushing. Navarra.

\begin{abstract}
Background. The aim of this work is to determine the situation of Cushing's Syndrome (CS) with respect to aetiology, diagnosis, treatment and cure in the last 25 years in Navarre.
\end{abstract}

Methods. Retrospective, observational study on 36 patients diagnosed with CS between 1985 and 2010.

Results. Eighty point five percent were women and 19.5\% were men. The average age on diagnosis was $39.3 \pm 13$ years The main clinical manifestations were: obesity $(85.3 \%)$, ful moon face (35.3\%) and myopathy (50\%). Aetiologically, in $58.3 \%$ the origin was hypophisiary, in $36.1 \%$ it was adrenal, and in $5.5 \%$ it was ectopic.

The UFC average was $343.5 \pm 310.1 \mu \mathrm{g} / 24$ hours. Serum cortisol after Nugent test was $15.6 \pm 8.6 \mu \mathrm{g} / \mathrm{dl}$. Low dose plasma cortisol average was $15.1 \pm 14.2 \mu \mathrm{g} / \mathrm{dl}$. There is a statistically significant $(\mathrm{p}<0.01)$ direct lineal correlation between UFC and the Nugent test, as well as between the Nugent test and low dose cortisol test. There are no statistically significant differences between adrenal and hypophisiary aetiology in either demographic factors or analytical results.

Treatment in all the hypophisiary and adrenal adenomas was surgical. Twenty-three point eight percent of the hypophisiaries required second surgery and subsequently radiotherapy (RT) and $9.5 \%$ only received RT following the first intervention. In the follow-up $52.9 \%$ were cured without sequels, $41.1 \%$ presented comorbidities and $5.9 \%$ died (without significant differences between adrenals and hypophisiaries).

Conclusion. The most frequent aetiology of CS was hypophisiary, which was more frequent in women. All of them are cured, although $41.1 \%$ presented comorbidities in the follow-up.

Key words. Cushing's syndrome. Navarre.
Servicio de Endocrinología. Complejo Hospitalario de Navarra

Recepción: 23 de abril de 2012

Aceptación provisional: 16 de mayo de 2012

Aceptación definitiva: 13 de junio de 2012

\section{Correspondencia:}

Leire Pérez García

Servicio de Endocrinología

Complejo Hospitalario de Navarra

Irunlarrea, 3

31008, Pamplona

E-mail: leyre.perez.garcia@navarra.es 


\section{INTRODUCCIÓN}

El exceso crónico de glucocorticoides se acompaña de un amplio rango de signos y síntomas conocidos como Síndrome de Cushing (SC) y se presenta de forma más frecuente secundariamente al uso iatrogénico de glucocorticoides ${ }^{1}$.

Etiológicamente, el SC puede ser ACTH (hormona adrenocorticotropa)-dependiente o ACTH-independiente. Las principales causas de SC ACTH-dependiente son: el adenoma hipofisario secretor de $\mathrm{ACTH}$ (enfermedad de Cushing) y el síndrome de ACTH ectópico (secreción de ACTH por tumores extrahipofisarios), que se asocia principalmente al carcinoma de células pequeñas pulmonar ${ }^{2}$. El SC ACTH-independiente se produce fundamentalmente por: adenomas adrenales, carcinomas adrenales, hiperplasia adrenal macronodular e hiperplasia adrenal micronodular ${ }^{1}$. La distribución etiológica por frecuencias del total de casos de SC es la siguiente: $65 \%$ enfermedad de Cushing, 7\% síndrome de ACTH ectópico, 18\% adenoma adrenal, $6 \%$ carcinoma adrenal, $3 \%$ hiperplasia adrenal macronodular y $1 \%$ hiperplasia adrenal micronodular ${ }^{1}$.

Las manifestaciones clínicas más sugestivas de SC son: estrías rojo-vinosas, plétora facial o cara de luna llena, debilidad muscular proximal, hematomas y osteoporosis sin causa justificada ${ }^{3}$. Frecuentemente, los pacientes con SC presentan manifestaciones clínicas que aparecen en la población general como obesidad troncular, depresión, diabetes mellitus, hipertensión arterial (HTA) o irregularidades menstruales ${ }^{3}$.

El diagnóstico inicial del SC es bioquímico, debiéndose realizar uno de los siguientes test: cortisol libre urinario (CLU), cortisol salival nocturno, test de supresión con $1 \mathrm{mg}$ de dexametasona (test de Nugent) o test de supresión débil con dexametasona ${ }^{3}$. Tras la confirmación del $\mathrm{SC}$, el siguiente paso es determinar si el $\mathrm{SC}$ es ACTH dependiente o independiente, para lo cual se determinará el nivel plasmático de ACTH: si es menor de $10 \mathrm{pg} /$ ml evidencia un SC ACTH-independiente ${ }^{1}$.
En este caso, la tomografía axial computarizada (TAC) de las glándulas adrenales es el siguiente paso en el diagnóstico. La gammagrafía con 131I-6 -iodometil-19norcolesterol se emplea en el diagnóstico diferencial del SC ACTH-independiente, objetivándose captación unilateral del radiotrazador en el adenoma adrenal, captación cortical bilateral y generalmente asimétrica en la hiperplasia macronodular y ausencia de captación bilateral en el carcinoma adrenal $^{4}$. En los pacientes con SC ACTH-dependiente se debe realizar una resonancia magnética (RM) hipofisaria con gadolinio ${ }^{1}$. El cateterismo bilateral de senos petrosos inferiores, se considera el gold standard y se recomienda en pacientes con SC ACTHdependiente con resultados discordantes en los estudios bioquímicos o radiológicos realizados ${ }^{5,6}$.

El objetivo de este estudio fue conocer la situación del SC en los últimos 25 años en la Comunidad Foral de Navarra, en cuanto a etiología, clínica, diagnóstico, tratamiento y curación, de forma que se puedan optimizar los recursos sanitarios para ofertar una mejor asistencia a estos pacientes.

\section{MATERIAL Y MÉTODOS}

Se estudiaron un total de 36 pacientes diagnosticados de SC en la Comunidad Foral de Navarra entre 1985 y 2010. Para la obtención de los datos se empleó la historia clínica informatizada y los archivos clínicos.

El diseño fue un estudio retrospectivo, observacional, tipo series de casos. Los datos fueron procesados con el programa estadístico SPSS versión 17.0.

Inicialmente, para el diagnóstico de hipercortisolismo se empleó el CLU en orina de 24 horas, que se considera altamente sugestivo de SC, confirmando prácticamente el diagnóstico si es cuatro veces mayor al valor máximo de referencia. En nuestro laboratorio, se emplea la técnica de quimioluminiscencia (Centaur XP Siemens ${ }^{\circledR}$ ) para la medición del CLU y del cortisol plasmático. Posteriomente se suele realizar el test de Nugent, que consiste en la administra- 
ción de 1mg de dexametasona vía oral a las 23 horas y determinación del cortisol sérico a las 8 am del día siguiente, pudiéndose excluir la presencia de hipercortisolismo con valores inferiores a $1,8 \mu \mathrm{g} / \mathrm{dl}^{3}$.

Actualmente, empleamos el cortisol salival nocturno (Salivette $($ ) ) medido en dos ocasiones entre las 23 y las 24 horas, mediante la técnica de ELISA (Enzyme Linked Inmunosorbent Assay) para el screening del SC. Este test tiene una sensibilidad del 92$100 \%$ y una especificidad del $93-100 \%$ para el despistaje del SC, existiendo una buena correlación entre el cortisol salival nocturno y la medición simultánea del cortisol sérico $^{3}$. Se considera especialmente útil en la población infantil y en el diagnóstico del $\mathrm{SC}$ cíclico. En nuestro laboratorio, se consideran valores superiores a $0,38 \mu \mathrm{g} / \mathrm{dl}$ como indicativos de probable SC. No se han incluido determinaciones del cortisol salival en el estudio estadístico de la muestra de pacientes seleccionada, por tratarse de un test de screening introducido recientemente en nuestro medio hospitalario.

En la mayoría de los casos $(88,2 \%)$ se practicó el test de frenado o supresión débil, que consiste en la administración de $0,5 \mathrm{mg}$ de dexametasona vía oral cada 6 horas durante dos días. Durante el segundo día se recoge orina de 24 horas para medición del CLU y el tercer día se mide el cortisol sérico a las $8 \mathrm{am}$. Se considera una respuesta normal, pudiéndose excluir de esa manera el hipercortisolismo, un CLU el segundo día $<10 \mu \mathrm{g} / 24 \mathrm{~h}$ y un cortisol sérico el tercer día $<1,8 \mu \mathrm{g} / \mathrm{dl}^{3}$.

El test de frenado o supresión fuerte, se empleó para completar el diagnóstico en aquellos casos de SC de origen hipofisario. Se utiliza fundamentalmente para realizar el diagnóstico diferencial entre enfermedad de Cushing (origen hipofisario) y síndrome de Cushing ACTH-dependiente de origen ectópico. Se administran $2 \mathrm{mg}$ de dexametasona vía oral cada 6 horas durante dos días. Durante esos días, se recoge orina de 24 horas para medición del CLU y a las 8 am del tercer día se cuantificó el cortisol sérico. En individuos con una respues- ta normal, el cortisol sérico y la ACTH el tercer día son indetectables, siendo el CLU $<5 \mu \mathrm{g} / \mathrm{dl}^{7}$. En pacientes con enfermedad de Cushing, el CLU tras frenado fuerte, debe reducirse un $90 \%$ respecto al CLU basal.

Para la realización del cateterismo de senos petrosos (realizado en el servicio de Radiología intervencionista), se insertó un catéter en ambos senos inferiores vía vena femoral, para medir el nivel de ACTH a nivel petroso o central y a nivel periférico antes de la inyección y a los 2 , 5 y 10 minutos tras la inyección de CRH. Se considera indicativo de enfermedad de Cushing un gradiente central/periférico $\geq 2$ antes de $\mathrm{CRH}$ y $\geq 3$ tras $\mathrm{CRH}^{8}$. $\mathrm{La}$ medición de ACTH se realiza mediante la técnica de quimioluminiscencia (Immulite 2000, Siemens ${ }^{\circledR}$ )

\section{RESULTADOS}

De los 36 pacientes estudiados: 29 $(80,5 \%)$ eran mujeres y 7 (19,5\%) hombres. La edad media al diagnóstico fue $39,3 \pm 13$ años (rango 17 a 68 años).

El principal motivo de consulta fue el fenotipo, siendo la manifestación clínica lo que motivó la derivación a consulta de Endocrinología en el 70,6\% de los pacientes. En el 8,8\% de los pacientes el motivo fue el hallazgo de un incidentaloma. En cuanto a la clínica al diagnóstico, el 85,3\% presentaban obesidad abdominal; el $76,5 \%$ cara de luna llena y el 50\% miopatía proximal. El 51,7 \% de las mujeres presentaban hirsutismo y el $31,03 \%$ alteraciones menstruales (Fig. 1). El 38,2 \% no presentaron HTA en ningún momento durante el seguimiento, el $35,3 \%$ de los pacientes tenían tratamiento antihipertensivo previo al diagnóstico de SC y el $26,5 \%$ presentaban HTA en el momento del diagnóstico. El $64,8 \%$ no presentaron diabetes mellitus (DM) en ningún momento, el $17,6 \%$ presentaron una glucemia basal alterada en algún momento del diagnóstico, el 14,7\% cumplían criterios diagnósticos de DM al diagnóstico, y el 2,9\% tenía una respuesta anormal en el test de tolerancia oral a la glucosa. 


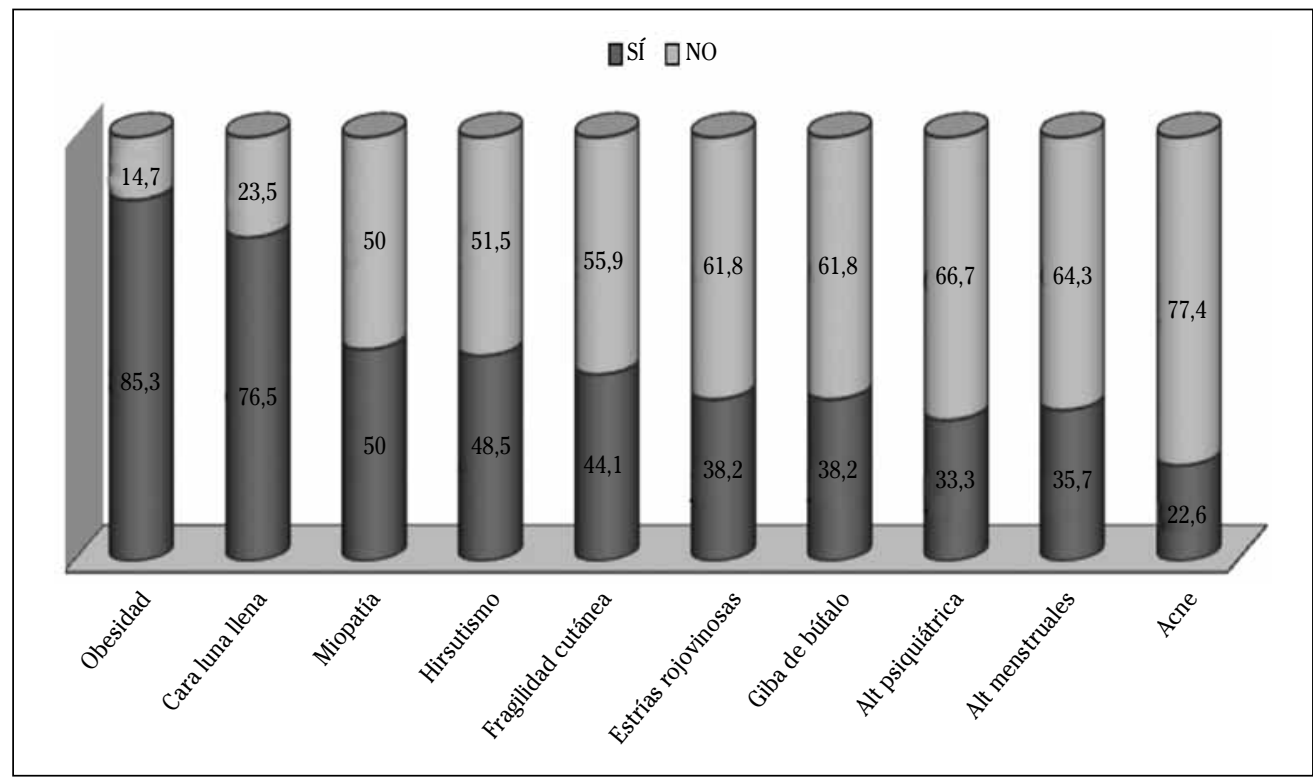

Figura 1. Manifestaciones clínicas al diagnóstico de síndrome de Cushing.

En veintiún (58,3\%) pacientes el origen del SC fue hipofisario (16 microadenomas y 5 macroadenomas). Trece pacientes (36,1\%) presentaron etiología adrenal (10 adenoma adrenal y 3 hiperplasia adrenal) y 2 pacientes $(5,5 \%)$ presentaron SC ectópico (secundario a tumor de células beta pancreáticas y tumor carcinoide pulmonar respectivamente) (Fig. 2). Durante la reali- zación del estudio estadístico, no se han incluido las determinaciones de analíticas de los dos pacientes que presentaron SC ectópico, por no considerarse oportuno. No se encontraron diferencias estadísticamente significativas entre adrenales e hipofisarios en cuanto a factores demográficos (edad y sexo) o resultados analíticos (CLU, Nugent y cortisol tras frenado débil).

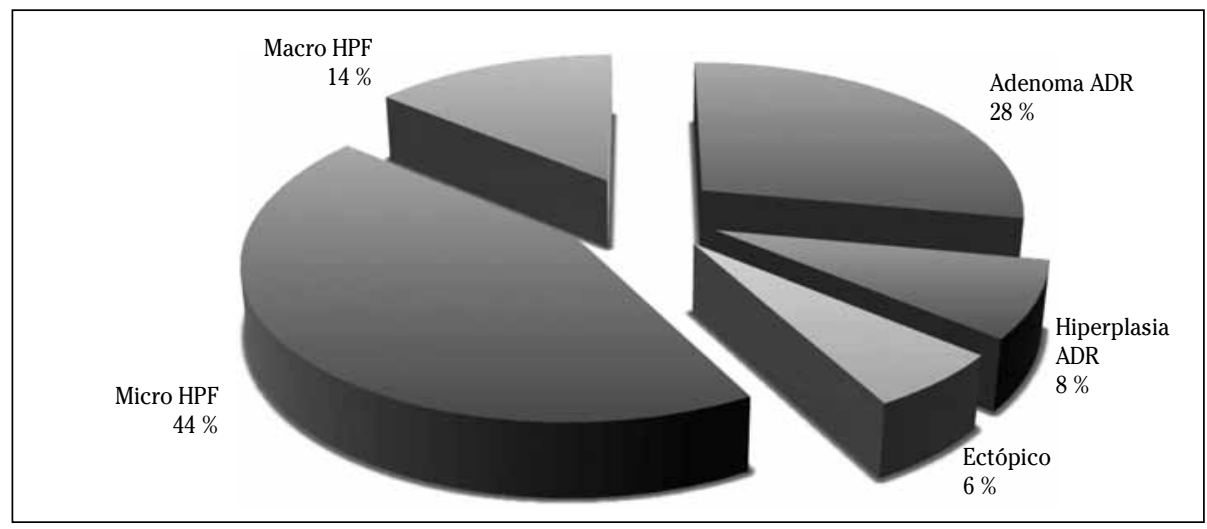

Figura 2. Etiología del síndrome de Cushing. HPF: hipofisario, ADR: adrenal, MACRO: macroadenoma, MICRO: microadenoma. 
La media del CLU fue $343,5 \pm 310,1 \mu \mathrm{g} / 24$ h (rango 48-1.464). Doce pacientes (35,3 \%) presentaron una determinación de CLU normal durante algún momento del diagnóstico. La media del cortisol sérico a las 23 horas fue de $21,8 \pm 13,12 \mu \mathrm{g} / \mathrm{dl}$ (rango 8,5-74). El $87 \%$ pacientes habían perdido el ritmo circadiano y $13 \%$ mantenían el ritmo circadiano intacto. Hemos considerado un valor de cortisol sérico nocturno a las 23 horas mayor de $7,5 \mu \mathrm{g} / \mathrm{dl}$, como reflejo de la pérdida del ritmo circadiano en nuestros pacientes con SC.

El cortisol sérico medio tras el test de Nugent fue de 15,6 $\pm 8,64 \mu \mathrm{g} / \mathrm{dl}$ (rango 3-37,1). La media de cortisol tras frenado

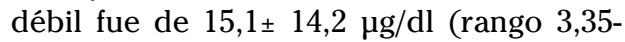
80). En ningún caso de los SC estudiados, el cortisol tras frenado débil o el cortisol en el test de Nugent fueron inferiores a $1,8 \mu \mathrm{g} /$ dl. El porcentaje de descenso de CLU tras frenado débil fue $32,62 \pm 48,42 \%$. Existe una correlación lineal directa estadísticamente significativa $(\mathrm{p}<0,01)$ entre CLU y el test de Nugent, así como entre Nugent y el cortisol tras el frenado débil.

El cortisol tras frenado fuerte se realizó en 22 pacientes siendo la media $16,68 \pm 22,9$ $\mu \mathrm{g} / \mathrm{dl}$ (rango1-89). El porcentaje de descenso del CLU fue de $27,6 \pm 78,7 \%$. El CLU tras frenado fuerte descendió por debajo del $90 \%$ en 6 pacientes y entre el 80 y el $90 \%$ en 3 pacientes.

En los 21 pacientes con enfermedad de Cushing, la media de ACTH fue 101,7 $\pm 84,5$ $\mathrm{pg} / \mathrm{ml}$ (rango 21-36). El tamaño medio de los adenomas hipofisarios fue 9,6 $699,6 \mathrm{~mm}$ (rango $1-40 \mathrm{~mm}$ ). El cateterismo de senos petrosos inferiores se realizó en 13 pacientes, 11 de ellos presentaron un gradiente central/ periférico $\geq 2$ antes de CRH y $\geq 3$ tras CRH. En cuanto a los 2 pacientes con gradientes inferiores al punto de corte, uno de ellos presentaba un drenaje venoso anómalo y el otro caso se trataba de una hiperplasia adrenal macronodular. A los 21 pacientes se le sometió a cirugía; en 20 de ellos vía transesfenoidal y en un caso vía transcraneal. El 66,6 \% se curaron en la primera intervención quirúrgica. Como segundo tratamiento en los 7 no curados, 5 se reintervinieron y posteriormente fueron tratados con radioterapia (RT) y 2 únicamente recibieron RT. A pesar de ello, 3 pacientes precisaron adrenalectomía bilateral por persistencia del hipercortisolismo.

El resultado anatomopatológico de los diez pacientes intervenidos de enfermedad de Cushing entre 2000 y 2010 fue de adenoma productor de ACTH, habiéndose realizado estudio de microscopía electrónica en el 50\% de estas muestras. En todos los pacientes la inmunohistoquímica fue positiva para ACTH, siendo también positiva para cromogranina y sinaptofisina en la mayoría de los pacientes estudiados. En aquellos pacientes en los que se había realizado estudio ultraestructural, habitualmente se apreciaban citoplasmas densamente granulares con abundantes gránulos densos. No se dispone de los resultados anatomopatológicos previos al año 2000.

Tras completar los tratamientos pertinentes, de los 21 pacientes de origen hipofisario, el $52,4 \%$ de los pacientes tienen déficit en un eje hipofisario, el 9,5\% en 2 ejes, el $4,8 \%$ en tres ejes y el $19 \%$ presentan déficits en cuatro o más ejes hipofisarios. En cuanto a la situación actual de los pacientes hipofisarios, el $52,4 \%$ de los pacientes están curados sin secuelas, los $38,1 \%$ curados con comorbilidades y el $9,5 \%$ han fallecido por causas ajenas al SC.

En los 13 pacientes con SC de origen adrenal, 10 fueron adenomas y 3 presentaban hiperplasia adrenal. El tamaño medio de los adenomas adrenales fue de $42,2 \pm 23,5$ mm (rango 20-85 mm). En los 10 pacientes que presentaban adenomas adrenales se practicó adrenalectamía uniltateral; 53,8\% vía subcostal y $23,1 \%$ vía laparoscópica. En los 3 pacientes $(23,1 \%)$ con hiperplasia adrenal, se realizó adrenalectomía bilateral. En todos ellos, se trataba de una hiperplasia macronodular, no habiéndose realizado estudio de expresión de receptores aberrantes en ninguno de los casos. Del total de trece pacientes con SC de origen adrenal, 7 (53,85\%) se curaron sin secuelas y $6(46,15 \%)$ se curaron presentando comorbilidades. Entre las principales comorbilidades cabe destacar las siguientes: miopatía, persistencia de la HTA y de la DM, alteraciones psiquiátricas y osteoporosis. 
Respecto a la evolución de las manifestaciones clínicas durante el seguimiento, el fenotipo fue la manifestación que presentó una mayor tasa de curación y la HTA la de menor porcentaje de curación.

Del conjunto de casos de SC, actualmente 18 pacientes (52,9\%) están curados sin secuelas, 14 pacientes (41,1\%) están curados pero con comorbilidades, y 2 pacientes $(5,9 \%)$ han fallecido.

\section{DISCUSIÓN}

Este estudio presenta las características clínicas, demográficas y terapéuticas de 36 pacientes diagnosticados de SC en los últimos 25 años en la Comunidad Foral de Navarra. En cuanto a la etiología el $58,3 \%$ de los pacientes presentaban un adenoma hipofisario, el $27,8 \%$ un adenoma adrenal y el 5,5\% de origen ectópico, datos similares a los obtenidos en el ERCUSYN (European Registry on Cushing's Syndrome), que proporciona datos del $66 \%$, el $27 \%$ y el $5 \%$ respectivamente ${ }^{9}$. El porcentaje de adenomas adrenales, parece ser superior al de estudios previos, que situaban esta etiología en torno a 5-22,3\%10. El ratio mujer/hombre en nuestra muestra es 5,8/1, algo superior al obtenido en el ERCUSYN de $4 / 1^{9}$.

Las principales manifestaciones clínicas al diagnóstico en nuestra serie fueron la obesidad $(85,3 \%)$, la cara de luna llena $(76,5 \%)$ y la miopatía proximal (50\%). El porcentaje de obesidad es similar al del registro ERCUSYN (81\%), mientras que las cifras de miopatía son mayores (67\%) en dicho registro ${ }^{9}$. En cuanto a las cifras de HTA, en nuestra serie estaba presente en el $61,8 \%$ de los pacientes, valores por debajo del $78 \%$ obtenido en el ERCUSYN ${ }^{9}$.

El tratamiento óptimo de la enfermedad de Cushing es la adenomectomía selectiva mediante microcirugía transesfenoidal, realizada por un neurocirujano experto ${ }^{10}$. En la enfermedad de Cushing, el índice de recurrencia aumenta con el paso del tiempo y cuando el cortisol plasmático en el postoperatorio inmediato es mayor de 2 $\mu \mathrm{g} / \mathrm{dl11}$. En nuestra serie, 5 microadenomas $(31,25 \%)$ tenían un valor de cortisol posto- peratorio inmediato $\leq 2 \mu \mathrm{g} / \mathrm{dl}$ y 10 microadenomas $(62,5 \%)$ tenían un valor $>2 \mu \mathrm{g} / \mathrm{dl}$, no habiéndose recogido dicho dato en un paciente. Trece de los 16 microadenomas se curaron en la primera cirugía, siendo el rango de cortisol postoperatorio entre los 3 microadenomas que recidivaron de 4-23 $\mu \mathrm{g} / \mathrm{dl}$. Únicamente un macroadenoma se curó en la primera intervención (20\%). El $80 \%$ precisó de una segunda intervención terapéutica, observándose tan solo en uno de los macroadenomas que recidivaron un cortisol plasmático postoperatorio de $1,4 \mu \mathrm{g} / \mathrm{dl}$. De modo que con un cortisol sérico postoperatorio $\leq 2 \mu \mathrm{g} / \mathrm{dl}$ la tasa de recurrencias de adenomas hipofisarios se situó en $4,76 \%$, similar al $3,77 \%$ obtenido en otros estudios ${ }^{11}$.

En la enfermedad de Cushing recurrente o persistente, repetir la cirugía transesfenoidal, se considera la primera opción terapéutica. En caso de que ésta fracase, la radiocirugía o la radioterapia convencional deben ser la segunda opción terapéutica ${ }^{10}$. La adrenalectomía bilateral está indicada en pacientes con hipercortisolismo persistente, a pesar de tratamiento médico con inhibidores de la esteroidogénesis enzimáticos adrenales (ketoconazol, metopirona, etomidato, mitotano, pasireótida...) o intolerancia a los mismos ${ }^{10}$. Así mismo, la adrenalectomía bilateral tiene un importante papel en el rápido control del hipercortisolimo en pacientes críticos ${ }^{12}$.

Los pacientes con enfermedad adrenal benigna se deben someter a adrenalectomía unilateral en el caso de los adenomas o bilateral en el caso de la hiperplasia micro o macronodular. En la actualidad, la vía laparoscópica es la principal vía de abordaje, ya que la vía transabdominal o la adrenalectomía retroperitoneal posterior abierta pueden causar mayor morbilidad ${ }^{13}$.

Las principales limitaciones del estudio realizado se producen como consecuencia de la ausencia de recogida de algunos datos al tratarse de un estudio retrospectivo. Además sería interesante completar el estudio con un nuevo estudio prospectivo incluyendo de manera sistemática la densitometría y un cuestionario de la calidad de vida del paciente con SC. 


\section{BIBLIOGRAFÍA}

1. Boscaro M, Arnaldi G. Approach to the Patient with possible Cushing's Syndrome. J Clin Endocrinol Metab 2009; 94: 3121-3131.

2. More J, Young J, Reznik Y, Raverot G, BorsonChazot F, Rohmer V et al. Ectopic ACTH syndrome in children and adolescents. J Clin Endocrinol Metab 2011; 96: 1213-1222.

3. Nieman LK, Biller BMK, Findling JW, NewellPrice J, Savage MO, Stewart PM et al. The diagnosis of Cushing's Syndrome: An Endocrine Society Clinical Practice Guideline. J Clin Endocrinol Metab 2008; 93: 1526-1540.

4. Yu-Erh H, Pei-Wen W, Hsu-Hua H, Wei-Jen C, Yun-Hsuan H, Yi-Chuan W et al. Role of 131-INP-59 adrenal imaging in patients of ACTHindependent Cushing's syndrome. Ann Nucl Med Sci 2001; 14: 75-83.

5. Arnaldi G, Angeli A, Atkinson AB, Bertagna X, Cavagnini F, Chrousos GP et al. Diagnosis and complications of Cushing's syndrome: a consensus statement. J Clin Endocrinolol Metab 2003; 88: 5593-5602.

6. Newell-Price J, Bertagna X, Grossman AB, NieMAN LK. Cushing's Syndrome. Lancet 2006; 367: 1605-1617.

7. NIEMAN LK. Establishing the cause of Cushing's Syndrome [sede web]. October
2, 2008. Uptodate Waltham, Massachusetts. http://www.uptodate.com/

8. Sharma S.T, Raff H, Nieman LK. Prolactin as a marker of successful catheterization during IPSS in patients with ACTH-dependent Cusshing's Syndrome. J Clin Endocrinol Metab 2011; 96: 3687-3694.

9. Valassi E, Santos A, Yaneva M, Tóth M, StrasburGER CJ, ChAnson P et al. The European registry on Cushing's Syndrome: 2-year experience. Baseline demographic and clinical characteristics. Eur J Endocrinol 2011; 165: 383-392.

10. Biller BMK, Grossman AB, Stewart PM, Melmed S, Bertagna X, Bertherat J et al. Treatment of adrenocorticotropin-dependent Cushing's Syndrome: A consensus statement. J Clin Endocrinol Metab 2008; 93: 2454-2462.

11. Ciric I, Zhao JC, DU H, Fidling JW, Molitch ME, WeIss RE et al. Transphenoidal surgery for Cushing disease. Experience with 136 patients. Neurosurgery 2012; 70: 70-81.

12. Tritos NA, Biller BMK, Swearingen B. Management of Cushing's disease. Nat Rev Endocrinol 2011; 7: 279-289.

13. Nieman LK. Treatment of Cushing's Syndrome: diminishing adrenal cortisol synthesis [sede web]. June 17, 2011. Uptodate Waltham, Massachusetts. http://www.uptodate.com/ 